\title{
Correction to: Comprehensive Integrated Digital Workflow to Guide Surgery and Prosthetics for Full-Arch Rehabilitation: A Narrative Review
}

Alessandro Pozzi, Lorenzo Arcuri, and Peter Moy

\section{Correction to: \\ Chapter 4 in: S. Jivraj (ed.), Graftless Solutions for the Edentulous Patient, BDJ Clinician's Guides, https://doi.org/10.1007/978-3-319-65858-2_4}

This book was inadvertently published with the incorrect author name in Chapter 4 as 'Lorenzi Arcuri'. This has now been amended throughout the book to the correct name as 'Lorenzo Arcuri'.

The updated online version of this chapter can be found at https://doi.org/10.1007/978-3-319-65858-2_4

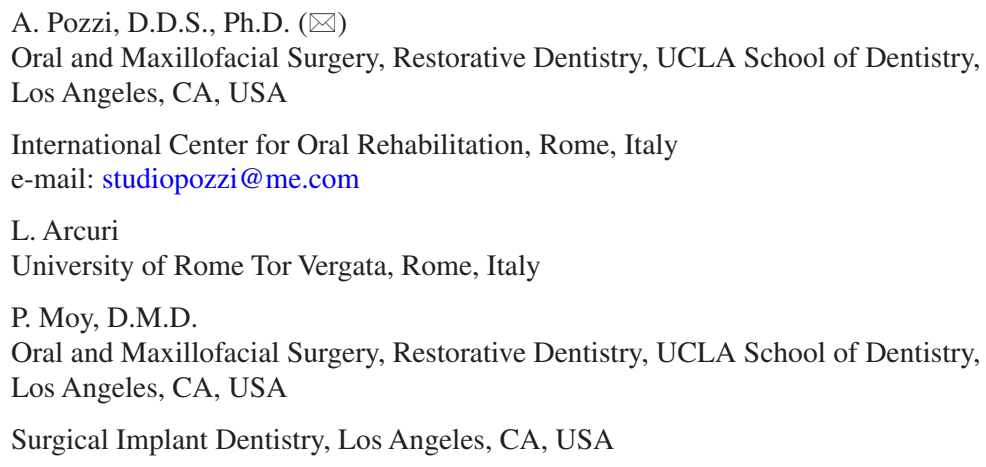

